\title{
Mental rotation task of hands: differential influence number of rotational axes
}

\author{
Arjan C. ter Horst $\cdot$ Rob van Lier $\cdot$ Bert Steenbergen
}

Received: 15 December 2009 / Accepted: 22 March 2010 / Published online: 8 April 2010

(C) The Author(s) 2010. This article is published with open access at Springerlink.com

\begin{abstract}
Various studies on the hand laterality judgment task, using complex sets of stimuli, have shown that the judgments during this task are dependent on bodily constraints. More specific, these studies showed that reaction times are dependent on the participant's posture or differ for hand pictures rotated away or toward the mid-sagittal plane (i.e., lateral or medial rotation, respectively). These findings point to the use of a cognitive embodied process referred to as motor imagery. We hypothesize that the number of axes of rotation of the displayed stimuli during the task is a critical factor for showing engagement in a mental rotation task, with an increased number of rotational axes leading to a facilitation of motor imagery. To test this hypothesis, we used a hand laterality judgment paradigm in which we manipulated the difficulty of the task via the manipulation of the number of rotational axes of the shown stimuli. Our results showed increased influence of bodily constraints for increasing number of axes of rotation. More specifically, for the stimulus set containing stimuli rotated over a single axis, no influence of biomechanical constraints was present. The stimulus sets containing stimuli rotated over more than one axes of rotation did induce the use of motor imagery, as a clear influence of bodily constraints on the reaction times was found. These findings extend and refine previous findings
\end{abstract}

A. C. ter Horst $(\triangle) \cdot$ R. van Lier $\cdot$ B. Steenbergen Donders Institute for Brain, Cognition and Behaviour, Centre for Cognition, Radboud University Nijmegen, Montessorilaan 3, P.O. Box 9104, 6500 HE Nijmegen, The Netherlands

e-mail: a.terhorst@donders.ru.nl

A. C. ter Horst · B. Steenbergen

Behavioural Science Institute,

Radboud University Nijmegen, Nijmegen, The Netherlands on motor imagery as our results show that engagement in motor imagery critically depends on the used number of axes of rotation of the stimulus set.

Keywords Mental rotation - Motor imagery $\cdot$ Visual imagery $\cdot$ Laterality judgment

\section{Introduction}

The mental rotation task is a well-established paradigm to study the cognitive process of mentally rotating objects. Typically, observed reaction time (RT) profiles show an increase in RT for increased angle of rotation indicating that participants mentally rotate stimuli to the upward position (Shepard and Metzler 1971; Parsons 1994; Jeannerod and Decety 1995). The mental rotation task was first studied by Shepard and Metlzer (1971). They used a task in which participants had to judge whether differently rotated 3D cube figures were identical or mirror reversed images of that figure (Shepard and Metzler 1971). It was found that RTs increased linearly with increasing angle of rotation. Other mental rotation studies used different paradigms in which the participants had to judge object or body part laterality, referred to as a laterality judgment task, first introduced by Sekiyama (1982). He found that RTs for judging hand laterality also increased with increased angles of rotation. However, RT did not increase linearly but quadratic and was not symmetrical about $180^{\circ}$ as is the case for $3 \mathrm{D}$ cube figures. This asymmetric quadratic RT profile was interpreted as evidencing kinesthetic influence of the laterality judgment of hands (Sekiyama 1982). It was postulated that participants engage in an embodied mental process in which biomechanical constraints influence the duration of the mental rotation process (Sekiyama 1982; Parsons 1987; 
Jeannerod 1994; Decety 1996a; Shenton et al. 2004; Sauner et al. 2006; Thayer and Johnson 2006). The influence of kinesthetic aspects or biomechanical constraints was further postulated by Parsons (1994). He showed that the time needed to actually rotate the own hand to an identical position as the displayed rotated hand corresponds to the time needed for a hand laterality judgment (Parsons 1987; Parsons 1994). More specifically, he showed that hand rotations away of the mid-sagittal plane (i.e., lateral rotation) resulted in larger RTs than hand rotation toward the mid-sagittal plane (i.e., medial rotation) for both overt as imagined movement. Thus, the RT profiles of imagined hand rotations were subject to the same biomechanical constraints experienced for overt movement. Laterality judgments of hands that are subject to biomechanical constraints make it conceivable that participants engage in embodied cognitive processing. This embodied processing is referred to as motor imagery (MI) (de Lange et al. 2006; de Lange et al. 2008). As a result, RT differences between lateral- and medial-rotated stimuli can be used as excellent measure to test for engagement in MI. When engaged in MI, participants mentally plan and perform a movement from a first person perspective without overtly performing the movement and without sensory feedback (Decety 1996a, b). The embodied nature of MI in a hand laterality judgment task was further evidenced by showing that RT profiles change as a consequence of changing the participant's posture (Parsons 1994; Sirigu and Duhamel 2001; de Lange et al. 2006; Helmich et al. 2007; Ionta et al. 2007; Ionta and Blanke 2009). Extending these findings, children at 5-7 years of age were also found to engage in MI in a hand laterality judgment task, possibly even more than adults (Funk et al. 2005). Visual imagery (VI), on the other hand, encompasses simulating executing a movement from a third person perspective (e.g., Steenbergen et al. 2007). Thus, VI is not subject to biomechanical constrains and as such not an embodied process.

Studies using hand laterality judgment tasks have not unequivocally shown to induce an MI strategy. As an example, we showed that participants with congenital alterations of posture on one side of the body, i.e., participants with hemiparetic cerebral palsy, lacked the presumed influence of posture on laterality judgments (Steenbergen et al. 2007). That is, it was hypothesized that these participants would show a different RT profile for laterality judgments of their affected versus their non-affected hand, but this was not evidenced by the data. In the study by Lust et al. (2006), groups of adults, healthy children and children suffering from developmental disorder were tested on their engagement in MI during a hand laterality judgment task with only hand stimuli shown from the back. Their results showed that RTs for both groups of children were subject to biomechanical constraints, as evident by the increased RT for difficult hand postures when compared to hand postures that are easier to adopt. However, the adult group showed no effect of biomechanical constraints and hence no engagement in MI (Lust et al. 2006).

We propose that the differences observed in literature on the effects of posture or, more general, biomechanical constraints on laterality judgments are due to the particulars of the stimulus set. The argument for this is following. In the studies of Lust et al. (2006) and our recent study, the stimulus set was largely the same. That is, all displayed pictures of hands were presented from a back view perspective in different angles of rotation. In contrast, studies showing strong effects of posture on RT used stimulus sets in which hands were presented from different viewpoints. See Table 1 for a short overview of literature on the hand laterality judgment task. Obviously, the use of these different perspectives from which the hand stimuli are presented yielded an increase in number of rotational axes and, as a consequence, an increase in overall task difficulty. Another factor that differed among studies and which may potentially cause the differential results is the number of rotational steps of the displayed stimuli, see Table 1 . However, the number of rotational steps appears to have only a marginal influence because in the study by Steenbergen et al. (2007) eighteen different rotational angles were used but no engagement in MI was found. Other studies, however, do show engagement in MI with as little as four angles of rotation (de Lange et al. 2006).

Given such diverging results, we hypothesize that the use of more rotational axes within a stimulus set facilitates MI engagement. The argument for this is that simple tasks, in which only in-plane-rotated back view hand stimuli are used (i.e., one axis of rotation), may promote the use of an alternative strategy based on the combination of finger and thumb orientation to solve the task (Lust et al. 2006). For more complex tasks, including for instance the use of both palm and back view hand stimuli, such a strategy will not suffice because one additional judgment on hand view is necessary next to judging hand laterality from the finger and thumb orientation. The inclusion of multiple rotational axes, and thus judgments, may therefore promote engagement in MI. That there is a neurophysiological difference between two-dimensional (2D) and three-dimensional (3D) rotation is already shown for three-dimensional objects. Kawamichi et al. (2007) showed that task difficulty enhanced by rotation dimensionality plays an important role in the selection of a motor strategy. They used threedimensional cube figures, similar to Shepard and Metzler (1971). Participants were presented a set of these figures rotated in 2D (i.e., in-plane) or 3D (i.e., in-plane and indepth). The results showed that the right superior parietal lobule was activated for 2D-rotated stimuli. In the case of 3D-rotated stimuli, the right dorsal premotor cortex (PMd) 
Table 1 Studies on hand laterality judgment task

\begin{tabular}{lllcl}
\hline Author & Year & View & Steps of rotation & MI engagement \\
\hline Ionta and Blanke & 2009 & Hands and feet, back, palm, thumb, little finger & 6 & Yes \\
de Lange et al. & 2008 & Back, palm & 7 & Yes \\
Ionta et al. & 2007 & As Ionta and Blanke (2009) & 18 & Yes \\
Steenbergen et al. & 2007 & Back & 8 & No \\
Helmich et al. & 2007 & Back, palm & 4 & Yes \\
de Lange et al. & 2006 & Back, palm & 8 & Yes \\
Lust et al. & 2006 & Back & 6 & Yes (children) \\
& & & 8 & No (adults) \\
Thayer and Johnson & 2006 & Back, palm & 4 & Yes \\
Sauner et al. & 2006 & Back, palm & 6 & Yes \\
Funk et al. & 2005 & Back, palm & 12 & Yes \\
Shenton et al. & 2004 & Back, palm & 12 & Yes \\
Parsons & 1994 & Back, palm, thumb, little finger, front finger, back palm & 8 & Yes \\
Parsons & 1987 & Back, palm, thumb, little finger, front finger, back palm & & Yes \\
Sekiyama & 1982 & Thumb, little finger, palm & & Yes \\
\hline
\end{tabular}

Note Studies on hand laterality judgment task and results on MI engagement. Both studies using only back view hand stimuli show no MI engagement (for adults). In contrast, studies using multiple viewpoints of hands within their stimulus set do show engagement in MI

was activated. These findings indicate that $2 \mathrm{D}$ and $3 \mathrm{D}$ rotation of cube figures activate different brain regions and may therefore be (partly) different neuronal or cognitive processes.

In the present study, we used three different conditions in which the number of axes of rotation increased from, respectively, only 1 axis to 3 axes of rotation. We expected an increasing difference in RTs between lateraland medial-rotated pictures of hands as a function of the number of axes of rotation. More specifically, for 1 axis of rotation, we expected to find no or marginal engagement in MI which will be evidenced by a marginal difference in RT between medial and lateral rotation, whereas increasing engagement in MI (i.e., increasing difference in RT between lateral- and medial-rotated hand pictures) is expected in the conditions with 2 - and 3 axes of rotation.

\section{Materials and methods}

\section{Participants}

Twelve healthy right-handed participants were included in the present study ( 8 women, age $22.5 \pm 3.7$ years, mean $\pm \mathrm{SD}$ ). All participants had normal or corrected-tonormal vision. No participant had a history of neurological or psychiatric disorder. The study was approved by the local ethics committee, and all participants gave written informed consent prior to the experiment, in accordance with the Helsinki declaration.
Stimuli

We used a custom made 3D hand model designed in a 3D image software package (Autodesk Maya 2009, USA). From this realistic model, we constructed all stimuli that were used in the experiments. All stimuli were displayed on a $19^{\prime \prime}$ LCD computer screen, at a distance of approximately $70 \mathrm{~cm}$ from the participants' eyes, resulting in a visual angle of approximately $6^{\circ}$.

The hand stimuli were rotated over three axes, resulting in three different rotational directions, namely: in-plane, longitudinal (referred to as 'view'), and in-depth, see Fig. 1. Three different sets of stimuli were used, namely: Set-1 containing only in-plane-rotated stimuli, Set- 2 with both in-plane- and longitudinal-rotated stimuli, and Set-3 containing in-plane-, longitudinal-, and in-depth-rotated stimuli. With $0^{\circ}$ of in-plane angular disparity as the upright position with the fingers pointing upward, see Fig. 1. Left and right hands were mirror images of each other but otherwise identical.

In Set-1, we used back view left and right hand stimuli in six different angles of in-plane rotation (i.e., $0^{\circ}, 60^{\circ}, 120^{\circ}$, $180^{\circ}, 240^{\circ}$, and $300^{\circ}$ ), resulting in 12 different stimuli, see upper left cell of Fig. 1. For Set-2, we used both back and palm view (i.e., two longitudinal rotational steps) stimuli of hands. All other aspects were identical to Set-1, resulting in 24 different stimuli, see upper row of Fig. 1. In Set-3, our set of stimuli were identical to the stimuli used in Set-2 but with three angles of in-depth rotations (i.e., $0^{\circ}, 60^{\circ}, 300^{\circ}$ ) with $0^{\circ}$ parallel to the vertical plane, resulting in 72 different stimuli, see Fig. 1. All stimuli were repeated three times 
Fig. 1 Shown are all used hand stimuli for Set-1: upper row, left column; Set-2: upper row; Set-3: all stimuli. Angles within stimuli represent in-plane angular disparity and angles displayed in the 'in-depth' column represent in-depth angular disparity

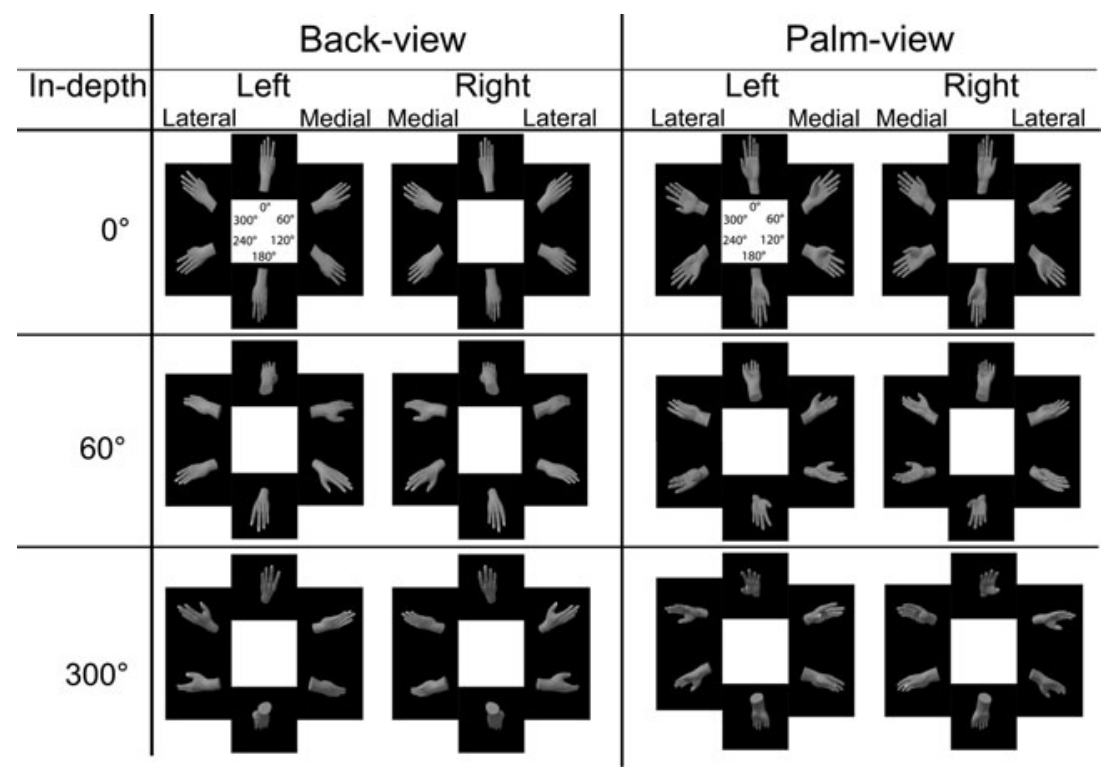

resulting in $36(12 * 3), 72(24 * 3)$ and $216(72 * 3)$ stimuli for Set-1, -2 , and -3 , respectively. All three conditions were preceded by a test of 24 stimuli to familiarize the participants with the task.

\section{Experimental procedure}

Participants were seated in a chair positioned in front of the computer screen. Stimuli were presented using customdeveloped software in Presentation (Neurobehavioral systems, Albany, USA). The displayed stimulus was visible until a response was given. Participants had to respond by pressing the left button with their left hand for left-hand stimuli and the right button with their right hand for righthand stimuli. Button responses and reaction times were digitally recorded for further analysis. After the response, a black screen was displayed. Participants were instructed to judge the laterality of the hand as fast and as accurate as possible, without explicit instructions on how to solve the task.

All stimuli were presented in seven sequential blocks with breaks in between. These seven blocks comprise: 1 block of Set-1 stimuli, 3 blocks of Set- 2 stimuli, and 3 blocks of Set-3 stimuli. All stimuli were repeated three times per Set. All Sets were presented blockwise and sequential, preventing mixing of blocks of the difficulty conditions over the experiment. The order of Sets were randomized and balanced across participants.

\section{Data analysis}

Reaction times smaller than $300 \mathrm{~ms}$ and larger than $3500 \mathrm{~ms}$ were excluded from analysis (total loss 3.2\% of trials); these upper and lower boundaries are based on similar studies using a hand laterality judgment task (Sekiyama
1987; Parsons 1994; Ionta et al. 2007; Iseki et al. 2008). Analysis was performed on correct responses. Incorrect responses were a 'left' response for a 'right' hand and vice versa and amounted to a total $6.4 \%$ of all trials. For analyses purposes, the different in-plane rotations were divided into medial- and lateral-rotated stimuli referred to as direction of rotation (DOR) in order to measure engagement in MI. Medial-rotated stimuli consisted of right hand $240^{\circ}$ and $300^{\circ}$ and left hand $60^{\circ}$ and $120^{\circ}$ in-plane-rotated stimuli. Lateral-rotated stimuli consisted of right hand $60^{\circ}$ and $120^{\circ}$ and left hand $240^{\circ}$ and $300^{\circ}$ in-plane-rotated stimuli. Data analysis was performed using repeated measures analysis of variance (ANOVA).

First, we analyzed the influence of increasing angle of rotation in order to test for the use of mental rotation for all sets. To this aim, we used an ANOVA with the following design: 2 within-subject factors (Set, Angle), with 3 levels for Set (Set-1, -2, and -3) and 4 levels for Angle $\left(0^{\circ}, 60^{\circ}\right.$, $120^{\circ}$, and $180^{\circ}$ ). With $60^{\circ}$ being the averaged value of both $60^{\circ}$ and $300^{\circ}$ in-plane-rotated stimuli and $120^{\circ}$ being the averaged value of both $120^{\circ}$ and $240^{\circ}$ in-plane-rotated stimuli. A significant effect for Angle with increasing RT with increased angle of rotation would indicate the use of mental rotation (Shepard and Metzler 1971). Then, we analyzed the effect of biomechanical constraints on the RTs by means of an ANOVA with the following design: 2 withinsubject factors (Set, DOR), with 3 levels for Set (Set-1, -2 and -3 ) and 2 levels for DOR (lateral rotation, medial rotation). A significant Set by DOR interaction would indicate that the influence of biomechanical constraints (as measured with DOR) differs between sets. This latter ANOVA design was also used to analyze the error data. Ad hoc analysis was Bonferroni corrected, and alpha level was set at $P=0.05$. 


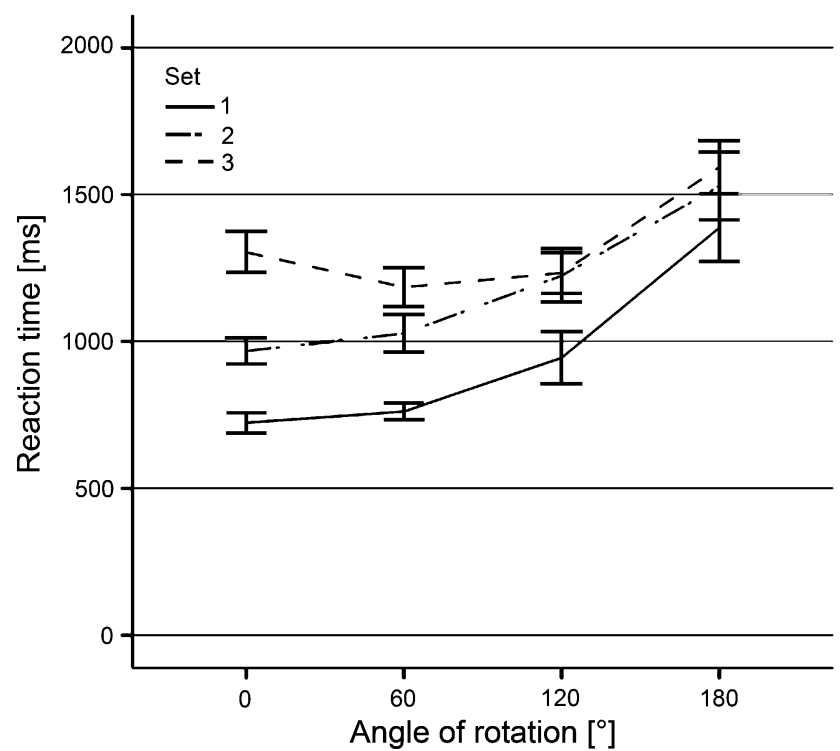

Fig. 2 Reaction times for all three sets, mirrored at $180^{\circ}$ (i.e., $60^{\circ}$ represents average RT for $60^{\circ}$ and $300^{\circ}$ rotated hand stimuli). Error-bars indicate standard error of the mean (SEM)

\section{Results}

The total number of incorrect responses (i.e., 6.4\% of all trials) corresponds to former studies (de Lange et al. 2006; Ionta et al. 2007). Between sets, the amount of erroneous responses differed significantly $[F(2,20)=4.692, P<0.05$, $\left.\eta^{2}=0.319\right]$. Within Set-1, Set-2, and Set-3, the amount of errors was 3.3, 4.6, and $7.3 \%$ of the number of trials per set, respectively. Set-1 differed significantly from Set-3 $(P<0.05)$. No significant effect of DOR nor an interaction was found in the error data.

The mental rotation analysis revealed a significant main effect for both Set $\left[F(2,22)=43.380, P<0.001, \eta^{2}=0.798\right]$ and Angle $\left[F(3,33)=38.772, P<0.001, \eta^{2}=0.779\right]$. Additionally, a significant interaction of Set by Angle $\left[F(6,66)=7.716, P<0.001, \eta^{2}=0.412\right]$ was found. Ad hoc analysis revealed a significant increase in RT with increasing angle of rotation for all three sets. Set- $1[F(3,33)=$ 37.732, $\left.P<0.001, \eta^{2}=0.774\right]$, Set $2[F(3,33)=20.941$, $\left.P<0.001, \eta^{2}=0.656\right]$, and Set-3 $[F(3,33)=28.789$, $\left.P<0.001, \eta^{2}=0.724\right]$. However, the mean RT for $0^{\circ}$ rotated stimuli in Set-3 was larger than the mean RT for $60^{\circ}$ rotated stimuli. This larger RT for $0^{\circ}$ was caused by the indepth-rotated hand stimuli. Overall, RTs increased significantly with increasing angle of rotation for all three sets, see Fig. 2.

The analysis on the influence of biomechanical constraints showed a significant main effect for Set $\left[F(2,22)=33.422, \quad P<0.001, \quad \eta^{2}=0.75\right] \quad$ and $\quad$ DOR

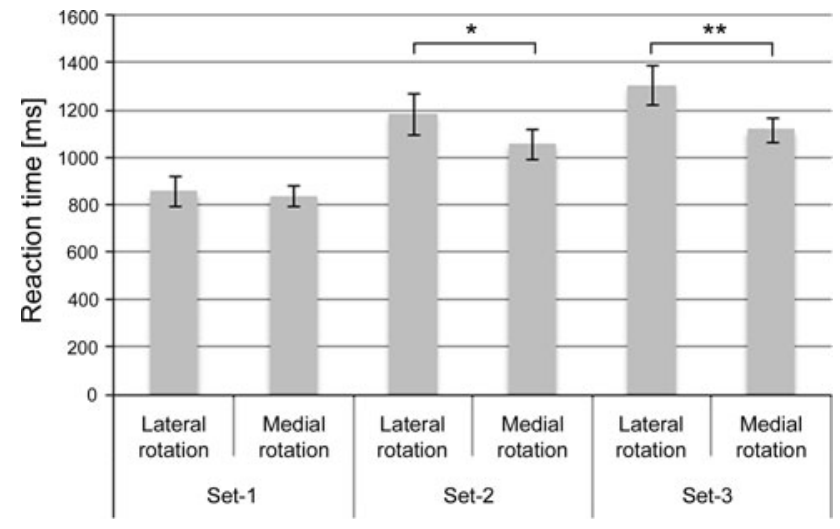

Fig. 3 Reaction times for all 3 Sets divided into lateral rotation and medial rotation. Lateral rotation indicates rotations away from the mid-sagittal plane, and medial rotation indicates rotations toward the mid-sagittal plane. As can be seen, the significant interaction of Set by DOR $(P<0.01)$ as represented by the differences in RTs between lateral and medial rotation (i.e., DOR) increases with increasing number of axes of rotation. * indicate significance at the $P<0.05$ level, ** indicate significance at the $P<0.01$ level. Error-bars indicate standard error of the mean (SEM)

$\left[F(1,11)=9.890, P<0.01, \eta^{2}=0.47\right]$. The Set effect was accounted for by the increase in RT for increasing number of axes of rotation, $848 \mathrm{~ms}, 1,123 \mathrm{~ms}$, and 1,214 ms for Set-1, Set-2, and Set-3, respectively. Set-1 differed significantly from both Set-2 and Set-3 $(P<0.001$ and $P<0.001$, respectively). Set- 2 and Set-3 did not differ significantly from each other $(P=0.168)$. The DOR effect was accounted for by the increased RT for laterally rotated stimuli $(1,117 \mathrm{~ms})$ compared to medially rotated stimuli $(1,006 \mathrm{~ms})$.

In order to support our hypothesis about increasing difference in RT between laterally and medially rotated stimuli, we expected a significant interaction between Set and direction of rotation (DOR). The ANOVA resulted in a significant interaction of Set by DOR $[F(2,22)=8.196$, $\left.P<0.01, \eta^{2}=0.43\right]$, which was accounted for by the increasing difference in RTs between laterally and medially rotated stimuli over the stimulus sets, resulting in significant simple DOR effects in Set-2 $[\mathrm{F}(1,11)=5.964$, $\left.P<0.05, \eta^{2}=0.35\right]$ and Set-3 $[F(1,11)=18.005, P<0.01$, $\left.\eta^{2}=0.62\right]$. Crucially, the simple DOR effect of Set-1 was not significant $(P=0.578)$, see Fig. 3 . The simple DOR effects differed significantly between Set-1 and Set-2 $\left[F(1,11)=6.276, P<0.05, \eta^{2}=0.363\right]$, and Set- 1 and Set-3 $\left[F(1,11)=13.344, P<0.01, \eta^{2}=0.548\right]$ but not between Set-2 and Set-3 $(P=0.198)$.

To control for the differences in mean RTs between the sets and its possible influence on the obtained DOR effects of the sets, we conducted an additional ANOVA with the data being normalized into the range of [0-1]. The ANOVA contained the following design: 2 within-subject factors (Set and DOR) with 3 levels for Set (Set-1, Set-2 
and Set-3) and 2 levels for DOR (lateral rotation, medial rotation). The results replicate the results with the non-normalized data except for an insignificant Set effect $(P=0.074)$. The lack of significant Set effect indicates that the normalization was effective as it reduced the differences in absolute value of the mean RTs between the sets.

As we only obtained a significant DOR effect in the sets including palm view stimuli, one might argue that only the palm view stimuli accounted for the obtained DOR effect in Set-2 and Set-3. To test whether a DOR effect is also present for in-plane-rotated back view stimuli (i.e., set-1 stimuli) within Set-2 and Set-3, we performed an ANOVA identical to the ANOVA testing the influence of biomechanical constraints, described in the 'Data analysis' section. However, we now only included RT values of the Set-1 stimuli in all sets. This test enables us to see whether a DOR effect is also present for back view stimuli in Set-2 and Set-3 and as a consequence enables us to examine whether only palm view or also back view stimuli induce the use of MI, depending on the context in which they are embedded. Additionally, this test shows whether participants used the same strategy for in-plane-rotated back view hand (i.e., Set-1) stimuli for all three sets or not.

The ANOVA revealed a significant main effect of Set $\left[F(2,22)=21.694, \quad P<0.001, \quad \eta^{2}=0.664\right] \quad$ and DOR $\left[F(1,10)=4.999, P<0.05, \eta^{2}=0.312\right]$ and an interaction of Set by DOR $\left[F(2,22)=3.429, P \leq 0.05, \eta^{2}=0.238\right]$. The interaction was accounted for by a lack of significant DOR effect for Set-1 stimuli in Set-1 $(P=0.578)$ and Set-2 $(P=0.237)$ but a significant DOR effect for Set-1 stimuli within Set-3 $\left[F(1,11)=8.620, P<0.05, \eta^{2}=0.439\right]$.

\section{Discussion}

In this study, we investigated the influence of multiple angles of rotation in the stimulus set in a hand laterality judgment task on participants' engagement in MI. We hypothesized that an increase in the number of axes of rotation facilitates an MI strategy. The number of axes of rotation cumulated from 1 (i.e., in-plane) to 2 and 3 axes over 3 separate stimulus sets.

According to the low error rates in all sets, which are well below chance level and the increasing RT in all sets for increasing angle of rotation (see Fig. 2), we may assume that participants did indeed mentally rotate the stimuli and were able to solve the mental rotation task accurately (Shepard and Metzler 1971; Sekiyama 1987; Parsons 1994; Helmich et al. 2007).

We showed increased RT differences between laterally and medially rotated hand stimuli (e.g., direction of rotation (DOR) effect) with increasing number of axes of rotation (see Fig. 3). Thus, there is an increasing influence of biomechanical constraints on RT with increasing number of axes of rotation, which exemplifies increased engagement in MI (Parsons 1994; Lust et al. 2006). In Set-1 (containing only in-plane-rotated back view stimuli), no effect of DOR was found. In contrast, both Set-2 (in-plane-rotated, backand palm view stimuli) and Set-3 (in-plane- and in-depthrotated, back- and palm view stimuli) revealed a significant DOR effect. The DOR effects in both the Set- 2 and Set- 3 conditions were accounted for by a smaller RT for medial compared to lateral rotations, which is in correspondence with literature (Parsons 1987; Parsons 1994). Additionally, the difference in obtained DOR effects between the sets was not accounted for by the differences in absolute mean RT between the sets as the analysis on the normalized data shows the same results. The process of MI is dependent on embodied cognitive processing and therefore affected by both the desired end-state and the current position of one's body (Jeannerod 1994; de Lange et al. 2008). In a mental rotation task of hands, the desired end-state is the presented hand posture. Because MI is subject to biomechanical constraints, RTs for lateral rotations of hands are prolonged compared to medial rotations if the participant engages in MI (Parsons 1987; Parsons 1994; Decety 1996a). Consequently, the presence of the DOR effect indicates the engagement in MI.

However, one might argue that participants memorized the Set-1 stimuli together with their laterality, leading to fast responses and no engagement in MI for Set-1. This explanation of the results is plausible because the Set-1 stimuli are seen in all three sets, therefore facilitating the memorizing of the stimuli. However, if participants indeed memorized the Set-1 stimuli, this would result in a lack of use of mental rotation at all. However, we showed a mental rotation process for Set-1 stimuli (Fig. 2), which was evident from the increase in RT with increasing angle of rotation. Hence, participants did not simply memorize Set- 1 stimuli.

Another possible explanation might be that the simple fact of adding palm view stimuli to a stimulus set results in the obtained difference in RT between medially and laterally rotated stimuli irrespective of the use of back view stimuli. The results indicate that we obtained this influence of biomechanical constraints only for the sets in which palm view stimuli were used. As a consequence, we sought to see whether the strategy used for the Set-1 stimuli (i.e., only in-plane-rotated back view stimuli) changes when they are embedded within a set of stimuli rotated around other rotational axes. The results indicate that the context in which the stimuli (i.e., Set-1 stimuli) are embedded is of influence on the strategy use, as the interaction of Set by DOR is significant. The changing effect of DOR over the sets indicates that the particulars of a stimulus set (i.e., number of rotational axes) influences 
the strategy used to solve the mental rotation task for the complete set and that strategies are not linked to a particular type of stimuli.

We expected an increase in RT from Set-1 till Set-3 because of the systematically adding of an extra rotational axis, thereby increasing the task difficulty. However, the general RT increase over all angles does not linearly increase from Set-1 to Set-3, as can be seen in both Figs. 2 and 3. This non-linear increase may be accounted for by two aspects. At first, the Set-1 stimuli were presented in a two-dimensional framework and the stimuli in both Set-2 and Set-3 were presented in a three-dimensional framework. It might be possible that, because of the dimensionality differences between the frameworks, the complexity increase is largest from Set-1 to Set-2, thereby introducing a larger overall increase in RT. Second, the non-linear increase may well be accounted for by the use of MI in both Set-2 and Set-3 and no MI in Set-1. This converges with findings of de Lange and colleagues, who showed that MI is a much slower strategy than the use of a third person's perspective (i.e., VI) (de Lange et al. 2005).

These findings beg the question as to what is the underlying cause of the observed differences in engagement in MI between the Sets? This question may be answered by examining the differences in DOR effects and the particulars of the stimuli between the three stimulus Sets. For the Set- 1 condition, it was sufficient for participants to judge the hand laterality by focusing on the combined finger and thumb orientation by, for example, determining whether an ' $L$ '-shape can be observed. If this is possible, then the presented hand must be a left hand or otherwise a right hand. This one possible strategy can be used for all angles of in-plane-rotated stimuli. However, when palm view stimuli are added, the use of that same strategy is not sufficient because, for an upright hand orientation, the same thumb orientation can lead to both left and right judgments due to the inverted view (i.e., thumb oriented to the left denotes a right back view hand or a left palm view hand). Therefore, an additional judgment needs to be incorporated into the strategy, namely a view judgment. It might be that the incorporation of multiple judgments into a strategy facilitates the use of the own body representation in order to judge the hand laterality. According to this suggested difference in strategy use, the inclusion of palm view stimuli within a stimulus set is crucial to induce MI engagement. The additional inclusion of in-depth rotation is likely to facilitate this engagement.

It has already been shown that humans are able to choose between two strategies in order to solve a mental rotation task (Kosslyn et al. 2001; Tomasino and Rumiati 2004). Studies on the hand laterality judgment task with children also show that there are two different strategies. At the same time, these studies showed that the implicit use of these different strategies differs between adults and children (Lust et al. 2006; Funk and Brugger 2008). Our data furthermore showed a dissociation between two strategies in adults. Collectively, it is likely that participants in our study use two different strategies to solve the mental rotation task, which encompasses a visually based strategy for Set-1 (as no influence of biomechanical constraints is evident) and a motor-guided strategy for Set-2 and Set-3.

In summary, this study shows that the number of axes of rotation of a stimulus set does critically influence the engagement in MI during a hand laterality judgment task. More specifically, combined use of palm and back view stimuli increases differences in RT between lateral and medial rotation compared to only presenting back view stimuli, implying a facilitated engagement in MI. Our results therefore show that participants do not automatically engage in MI in a hand laterality judgment task, but that engagement is critically dependent on the set of stimuli used. A simple set of stimuli might result in developing and using a strategy that lacks MI engagement, whereas a more difficult set of stimuli promotes engagement in MI. These results have implications for generalization of results of different studies on the hand laterality judgment task and may explain some of the differences observed in MI engagement between studies.

These results have implications for clinical applications. In several studies, motor imagery is used for rehabilitation purposes by, for instance, patients with stroke (Braun et al. 2006; Sharma et al. 2006), patients with Parkinson's disease and patients with complex regional pain syndrome (see Dickstein and Deutsch 2007 for a review). A major challenge in these studies is to engage participants into the use of motor imagery. Only then similar brain networks are active as in actual motor control, which enhances motor recovery. One way to establish this is by only including participants that are able to use motor imagery. Validated questionnaires such as the Movement Imagery Questionnaire (MIQ) can be used for this purpose. In addition, the use of a task that implicitly induces the use of a mental movement, such as the hand laterality judgment task, would probably facilitate the patient's ability to engage in a mental movement as this engagement does not depend on the conscious effort of the patient in imagining the movement. Finally, our results show that a hand laterality judgment task must be designed such that participants do not develop a strategy based on the visual characteristics of the stimuli.

Open Access This article is distributed under the terms of the Creative Commons Attribution Noncommercial License which permits any noncommercial use, distribution, and reproduction in any medium, provided the original author(s) and source are credited. 


\section{References}

Braun SM, Beurskens AJ, Borm PJ, Schack T, Wade DT (2006) The effects of mental practice in stroke rehabilitation: a systematic review. Arch Phys Med Rehabil 87:842-852

de Lange FP, Hagoort P, Toni I (2005) Neural topography and content of movement representations. J Cogn Neurosci 17:97-112

de Lange FP, Helmich RC, Toni I (2006) Posture influences motor imagery: an fMRI study. Neuroimage 33:609-617

de Lange FP, Roelofs K, Toni I (2008) Motor imagery: a window into the mechanisms and alterations of the motor system. Cortex 44:494-506

Decety J (1996a) Do imagined and executed actions share the same neural substrate? Brain Res Cogn Brain Res 3:87-93

Decety J (1996b) Neural representations for action. Rev Neurosci 7:285-297

Dickstein R, Deutsch JE (2007) Motor imagery in physical therapist practice. Phys Ther 87:942-953

Funk M, Brugger P (2008) Mental rotation of congenitally absent hands. J Int Neuropsychol Soc 14:81-89

Funk M, Brugger P, Wilkening F (2005) Motor processes in children's imagery: the case of mental rotation of hands. Dev Sci 8:402-408

Helmich RC, de Lange FP, Bloem BR, Toni I (2007) Cerebral compensation during motor imagery in Parkinson's disease. Neuropsychologia 45:2201-2215

Ionta S, Blanke O (2009) Differential influence of hands posture on mental rotation of hands and feet in left and right handers. Exp Brain Res 195:207-217

Ionta S, Fourkas AD, Fiorio M, Aglioti SM (2007) The influence of hands posture on mental rotation of hands and feet. Exp Brain Res 183:1-7

Iseki K, Hanakawa T, Shinozaki J, Nankaku M, Fukuyama H (2008) Neural mechanisms involved in mental imagery and observation of gait. Neuroimage 41:1021-1031

Jeannerod M (1994) The representing brain: neural correlates of motor intention and imagery. Behav Brain Sci 17:187-245

Jeannerod M, Decety J (1995) Mental motor imagery: a window into the representational stages of action. Curr Opin Neurobiol 5:727732
Kawamichi H, Kikuchi Y, Noriuchi M, Senoo A, Ueno S (2007) Distinct neural correlates underlying two- and three-dimensional mental rotations using three-dimensional objects. Brain Res 1144:117-126

Kosslyn SM, Ganis G, Thompson WL (2001) Neural foundations of imagery. Nat Rev Neurosci 2:635-642

Lust JM, Geuze RH, Wijers AA, Wilson PH (2006) An EEG study of mental rotation-related negativity in children with developmental coordination disorder. Child Care Health Dev 32:649-663

Parsons LM (1987) Imagined spatial transformations of one's hands and feet. Cogn Psychol 19:178-241

Parsons LM (1994) Temporal and kinematic properties of motor behavior reflected in mentally simulated action. J Exp Psychol Hum Percept Perform 20:709-730

Sauner D, Bestmann S, Siebner HR, Rothwell JC (2006) No evidence for a substantial involvement of primary motor hand area in handedness judgements: a transcranial magnetic stimulation study. Eur J Neurosci 23:2215-2224

Sekiyama K (1982) Kinesthetic aspects of mental representations in the identification of left and right hands. Percept Psychophys 32:89-95

Sekiyama K (1987) Mental rotation of kinesthetic hand images and modes of stimulus presentation. Shinrigaku Kenkyu 57:342-349

Sharma N, Pomeroy VM, Baron JC (2006) Motor imagery: a backdoor to the motor system after stroke? Stroke 37:1941-1952

Shenton JT, Schwoebel J, Coslett HB (2004) Mental motor imagery and the body schema: evidence for proprioceptive dominance. Neurosci Lett 370:19-24

Shepard RN, Metzler J (1971) Mental rotation of three-dimensional objects. Science 171:701-703

Sirigu A, Duhamel JR (2001) Motor and visual imagery as two complementary but neurally dissociable mental processes. J Cogn Neurosci 13:910-919

Steenbergen B, van Nimwegen M, Craje C (2007) Solving a mental rotation task in congenital hemiparesis: motor imagery versus visual imagery. Neuropsychologia 45:3324-3328

Thayer ZC, Johnson BW (2006) Cerebral processes during visuomotor imagery of hands. Psychophysiology 43:401-412

Tomasino B, Rumiati RI (2004) Effects of strategies on mental rotation and hemispheric lateralization: neuropsychological evidence. J Cogn Neurosci 16:878-888 